\title{
Bur open Dietary patterns and colorectal cancer recurrence and survival: a cohort study
}

\author{
Yun Zhu, ${ }^{1,2}$ Hao Wu, ${ }^{1}$ Peizhong Peter Wang, ${ }^{1,2}$ Sevtap Savas, ${ }^{3,4}$ \\ Jennifer Woodrow, ${ }^{1}$ Tyler Wish, ${ }^{3}$ Rong Jin, ${ }^{5}$ Roger Green, ${ }^{3}$ Michael Woods, ${ }^{3}$ \\ Barbara Roebothan, ${ }^{1}$ Sharon Buehler, ${ }^{1}$ Elizabeth Dicks, ${ }^{6}$ John R Mclaughlin, ${ }^{7}$ \\ Peter T Campbell, ${ }^{8}$ Patrick S Parfrey ${ }^{6}$
}

To cite: Zhu Y, Wu H, Wang PP, et al. Dietary patterns and colorectal cancer recurrence and survival: a cohort study. BMJ Open 2013;3:e002270. doi:10.1136/bmjopen-2012002270

- Prepublication history for this paper are available online. To view these files please visit the journal online (http://dx.doi.org/10.1136/ bmjopen-2012-002270).

$\mathrm{YZ}$ and HW contributed equally to this study.

Received 26 October 2012 Revised 17 October 2012 Accepted 19 December 2012

This final article is available for use under the terms of the Creative Commons Attribution Non-Commercial 2.0 Licence; see http://bmjopen.bmj.com

For numbered affiliations see end of article.

Correspondence to Dr Peizhong Peter Wang; pwang@mun.ca

\section{ABSTRACT}

Objective: To examine the association between dietary patterns and colorectal cancer (CRC) survival.

Design: Cohort study.

Setting: A familial CRC registry in Newfoundland.

Participants: 529 newly diagnosed CRC patients from Newfoundland. They were recruited from 1999 to 2003 and followed up until April 2010.

Outcome measure: Participants reported their dietary intake using a food frequency questionnaire. Dietary patterns were identified with factor analysis. Multivariable Cox proportional hazards models were employed to estimate $\mathrm{HR}$ and $95 \% \mathrm{Cl}$ for association of dietary patterns with CRC recurrence and death from all causes, after controlling for covariates.

Results: Disease-free survival (DFS) among CRC patients was significantly worsened among patients with a high processed meat dietary pattern (the highest vs the lowest quartile HR 1.82, $95 \% \mathrm{Cl} 1.07$ to 3.09). No associations were observed with the prudent vegetable or the high-sugar patterns and DFS. The association between the processed meat pattern and DFS was restricted to patients diagnosed with colon cancer (the highest vs the lowest quartile: $\mathrm{HR} 2.29,95 \% \mathrm{Cl} 1.19$ to 4.40) whereas the relationship between overall survival (OS) and this pattern was observed among patients with colon cancer only (the highest vs the lowest quartile: HR $2.13,95 \% \mathrm{Cl} 1.03$ to 4.43$)$. Potential effect modification was noted for sex ( $p$ value for interaction 0.04, HR 3.85 for women and 1.22 for men).

Conclusions: The processed meat dietary pattern prior to diagnosis is associated with higher risk of tumour recurrence, metastasis and death among patients with CRC.

\section{INTRODUCTION}

Colorectal cancer (CRC) is the third most frequent cancer and the second leading cause of cancer death in Canada. Epidemiological studies have established a strong link between a few dietary factors, such as fibre (inversely) and red/processed meat (increases risk), and the risk of developing CRC, ${ }^{2}$ although most studies have

\section{ARTICLE SUMMARY}

Article focus

- We used the data of 529 colorectal cancer patients in Newfoundland and Labrador to investigate the association of dietary patterns and colorectal cancer survival.

- We further explored if the relationship between dietary pattern and colorectal cancer survival is modified by sex, physical activity and BRAF mutation.

\section{Key messages}

- The processed meat dietary pattern is associated with a worsened colorectal cancer disease-free survival.

- The prudent vegetable or the high-sugar patterns show no association with disease-free survival.

- The relationship between processed meat pattern and colorectal cancer survival is modified by sex.

Strengths and limitations of this study

- The sample size is reasonably large with detailed information on diet, lifestyle and molecular characteristics.

- Recall bias remains a problem since food consumption was collected from1 year prior to colorectal cancer diagnosis. In addition, dietary patterns only reflect food consumption before diagnosis which might be modified after diagnosis.

focused primarily on individual foods or nutrients. Since foods and nutrients act synergistically rather than in isolation, ${ }^{3-6}$ recent research has investigated the role of dietary patterns on CRC incidence. Dietary patterns identified in prior research often include 'Western' and 'prudent' patterns. Adherence to the Western diet pattern, characterised by high intakes of meat, fat, sweets and desserts, is often associated with increased risk of CRC $^{5-9}$ whereas strong adherence to the prudent pattern, characterised by high intakes of fruit, vegetable, fish and poultry, often shows an inverse ${ }^{78}$ or null ${ }^{5610}$ association with CRC risk. 
The highest CRC incidence and death rates in Canada are observed in the province of Newfoundland and Labrador (NL). ${ }^{1}$ Geographically isolated in the Atlantic Ocean, NL has long maintained its traditional foods, a Western-style diet consisting of a large proportion of processed meat, red meat and insufficient vegetables. ${ }^{11}$ Several studies have partially attributed the high CRC incidence rate in NL to its unique diet, ${ }^{11-13}$ but no study has explored the association between the NL diet and its impact on survival among CRC patients.

This prospective cohort study investigated the influence of dietary patterns, identified by factor analysis, on survival and recurrence or metastasis among an incident case series of 529 CRC patients from NL. In addition, the present study evaluated possible effect modification among dietary patterns with gender, physical activity and tumour molecular phenotype.

\section{SUBJECTS AND METHODS}

\section{Study participants}

Patients in this prospective cohort study were enrolled through the Newfoundland Familial Colorectal Cancer Registry, described in detail elsewhere. ${ }^{14}{ }^{15}$ In brief, during the time period from 1999 to 2003, patients aged $20-75$ years, newly diagnosed with pathologically confirmed, invasive CRC were eligible for inclusion in the study (International Classification of Diseases (ICD)-9 codes: $153.0-153.9,154.0-154.3$ and 154.8 or ICD-10 codes: $18.0-18.9,19.9$ and 20.9).

Written, informed consent was required from each study participant to access their archived tumour tissue and medical records. If patients died before they could give consent (the median time from date of diagnosis to date of consent was 1.8 years), a close relative/proxy, who has lived with the patient, was invited to participate. Enrolling deceased cases through proxies could remove the potential bias of eliminating patients at a late distant stage. ${ }^{14}$ Thus, the inception cohort consisted of 750 eligible patients $(64 \%)$.

Consenting participants completed and returned a detailed food frequency questionnaire (FFQ), personal history questionnaire (PHQ) and family history questionnaire (FHQ). All questionnaires were self-completed. Assistance from study staff was available to help with understanding items on the questionnaires. To capture additional cancer diagnosis or recurrence in the family after enrolment, the FHQ was distributed to participants for the second time midway through the follow-up. To be included in this analysis, patients had to have completed at least the FFQ provided informative lifestyle and medical data from the PHQ and had known vital status information by the end of the follow-up period (April 2010). For patients who died prior to enrolment, the designated relative/proxy completed the aforementioned questionnaires. The final analytical cohort comprised of 529 eligible participants. The study protocol was approved by the Human Investigation Committee of Memorial University of Newfoundland.

\section{Dietary assessment and food grouping}

Diet was assessed using a semiquantitative FFQ developed from the well-known Hawaii FFQ ${ }^{16}$ on the basis of a validated instrument adapted for the Canadian population. ${ }^{17} 18$ The FFQ included 170 foods, beverages, and vitamin supplements and dietary supplements. ${ }^{19}$ Foods indigenous to the Newfoundland population (eg, salted/pickled meat and smoked/pickled fish) were also included. For each food item or beverage, the participants were asked to estimate their frequency of consumption and usual portion size as 'Small', 'Regular' or 'Large' 1 year prior to their colon or rectal cancer diagnosis. Portion sizes for specific foods were depicted in photographs. Nutrient and total energy intakes were calculated by multiplying the frequency of consumption of each food by the nutrient content of the portion size based on the composition values from the 2005 Canadian Nutrient file. ${ }^{12}$ Taking a similar grouping scheme to that used elsewhere ${ }^{3}$ we collapsed individual food items on the FFQ into 39 predefined food groups based on the roles of food in diet and cancer aetiology. Distinct food items were reserved as individual categories if it was deemed inappropriate to combine them (eg, jam, pies, beer and wine).

\section{Covariates}

Sociodemographic data, such as age, sex, marital status and education attainment, were gathered by the selfadministered PHQ. The PHQ also included items regarding medical history, bowel screening history, physical activity, reproductive factors (women only) and alcohol and tobacco use. Family history of cancer was assessed by the FHQ.

\section{Study outcomes}

Study outcomes were ascertained from follow-up questionnaires, local newspapers (eg, death notices), death certificates, autopsy, pathology, radiology, surgical reports, as well as physician's notes. Additional data were gathered from the Dr H Bliss Murphy Cancer Care Foundation and Statistics Canada. ${ }^{20}$ The cause of death was obtained for 93 of 168 deceased patients in this cohort, classified according to the ICD codes for underlying or contributing cause of death; ${ }^{21}$ the majority $(91 \%)$ of these had died from CRC. Since specific cause of death was not available for all deceased participants, all-cause mortality was used for analysis. In this study, two endpoints were considered: the first was disease-free survival (DFS), defined as time from cancer diagnosis to the first confirmed tumour recurrence, metastasis or death from all causes occurring up to April 2010; the second end point was overall survival (OS), measured from the date of cancer diagnosis to the date of death from all causes. Patients who did not have an event by 
the end of the follow-up were censored at the date of last contact.

\section{Molecular assessment}

The p.V600E BRAF mutation and (microsatellite instability) MSI status for the tumour DNA have been determined in previous studies using standard protocols. ${ }^{22-24}$ Briefly, the mutational hotspot c.1799T $>$ A. (p. Val600Glu) in the $B R A F$ gene was detected using $B R A F$ V600E allele-specific primers, with controls amplifying the GAPDH gene. ${ }^{24}$ Positive mutations were then verified by direct automatic sequencing. ${ }^{24}$ For MSI analyses, a panel of 10 microsatellite repeats (BAT25, BAT26, BAT40, BAT34C4, D5S346, D17S250, ACTC, D18S55, D10S197 and MYCL) were used to amplify both tumour and normal DNA. ${ }^{22} 23$ MSI status was defined as MSI-high if $30 \%$ or more of the markers were unstable and MS-stable/MSI-low, if less than $30 \%$ of the markers showed instability. ${ }^{25}{ }^{26}$ The primer sequences and PCR conditions are provided in detail in earlier studies from this cohort. ${ }^{14} 22-24$

\section{Statistical analysis}

Exploratory principal component factor analysis ${ }^{27}$ was used to identify major dietary patterns based on 39 predefined food groups from the FFQ. A varimax rotation (orthogonal) procedure was applied to rotate these factors, meaning that it produces uncorrelated, easy interpreted components that explain the greatest amount of variance in the original food groups. ${ }^{28}$ We determined the number of factors to retain for interpretation on the basis of criteria as follows: factor eigenvalue greater than 1.15 , the scree plot, the proportion of variance explained and factor interpretability. ${ }^{9}$ Patterns were labelled based on food groups with absolute rotated factor loading matrix greater than or equal to 0.50. Each participant was assigned a factor score for each pattern (factor) by summing the intakes from each food group multiplied by optimal weights (factor loadings). ${ }^{5}$ Individuals with a higher factor score had a closer adherence to that pattern. ${ }^{5}$

Comparisons for baseline characteristics across quartiles of dietary patterns were performed using the ANOVA test for continuous variables and $\chi^{2}$ test for categorical variables. Cox proportional hazards models, each adjusting for energy intake and critical covariates, were used to evaluate the association between individual dietary pattern and CRC recurrence and mortality, represented by HR and 95\% CI. Potential confounders were assessed by the log-rank test in a univariate setting; those with the $p$ value less than 0.1 were considered for inclusion. The final models only retained the items that entered the models at $\mathrm{p}<0.1$ or altered the effect estimates by $10 \%$ or more; these include sex, age at diagnosis, stage at diagnosis, body mass index (BMI), marital status, family history, reported screening procedure, reported chemoradiotherapy and MSI status. All models were run with the adjustment for total energy intake by including total calories in the model. The assumption of proportional hazard rates was verified by checking the parallelism of the Kaplan-Meier curves and by including time-dependent covariates in the models to test for statistical significance. ${ }^{29}$ Statistical linear trend was examined by modelling the median value of each quartile as an ordinal variable in a linear regression. ${ }^{5}$ Potential interactions were evaluated by comparing estimates from stratified analyses and testing significance of interaction terms with a Wald test. ${ }^{5}$

A sensitivity analysis was implemented by eliminating stage-advanced patients enrolled through proxies and recalculating survival time from the completion of the first questionnaire to a predefined event, in order to determine whether associations might vary with the exclusion of stage-advanced cancer. Statistical significance was accepted for two-sided $\mathrm{p}<0.05$. All data management and analyses were performed with SAS software V.9.2 (SAS Institute Inc, Cary, North Carolina, USA).

\section{RESULTS}

The cohort was followed for a median of 6.4 years (minimum 1.3 years; maximum 10.9 years). A total of 168 patients died from all causes and 30 had a cancer recurrence or metastasis by the end of study follow-up (April 2010).

\section{Dietary patterns}

Three distinct dietary patterns, labelled 'processed meat pattern', 'prudent vegetable pattern' and 'high-sugar pattern', were extracted using the aforementioned factor analysis procedure. These patterns explained $73.82 \%$ of total variance in the original 39 food groups (table 1). A higher factor loading matrix of a given food group is representative of a greater contribution of that food group on that specific pattern. Therefore, the first pattern, termed 'processed meat', was characterised by higher loadings and thus higher consumptions of cured/processed meat, cured/processed red meat, red meat, fish and processed fish; the second pattern, labelled 'prudent vegetable', displayed higher loadings on other greens, other fruit, other vegetables and tomato sauce; and the third pattern, named 'high sugar', showed higher loadings on desserts and sweets, pies and tarts.

\section{Baseline characteristics by quartiles of dietary patterns}

Higher processed meat pattern scores at baseline were detected in men, ever smokers, patients who were single and individuals who had higher BMI at the time of diagnosis (table 2). Higher prudent vegetable pattern scores were observed in women, never smokers, those with a slightly later age of diagnosis and with patients who had a tumour harbouring the $p$.V600E BRAF mutation. None of these characteristics varied significantly by quartiles of high-sugar pattern scores. 
Table 1 Factor loadings and explained variances (VAR) for the three major dietary patterns identified from the food frequency questionnaire at baseline using a principal component factor analysis, Newfoundland

\begin{tabular}{|c|c|c|c|}
\hline Food groups & Processed meat pattern & Prudent vegetable pattern & High-sugar pattern \\
\hline Milk & - & 0.19 & - \\
\hline Yogurt & - & 0.31 & - \\
\hline Sugar & - & -0.19 & 0.20 \\
\hline Tea & - & - & 0.17 \\
\hline Coffee & 0.17 & - & - \\
\hline Soft drinks & 0.19 & - & - \\
\hline Cheese & 0.15 & 0.21 & - \\
\hline Egg & 0.21 & - & 0.16 \\
\hline Mixed dishes & 0.31 & 0.17 & 0.23 \\
\hline Red meat & 0.69 & - & 0.17 \\
\hline Cured/processed red meat & 0.73 & - & 0.21 \\
\hline Cured/processed meat & 0.93 & - & - \\
\hline Game & 0.23 & - & - \\
\hline Poultry & 0.22 & 0.27 & - \\
\hline Fish & 0.58 & 0.32 & -0.22 \\
\hline Processed fish & 0.50 & 0.25 & - \\
\hline Fruit juice & - & 0.24 & 0.23 \\
\hline Root vegetables & 0.28 & - & 0.15 \\
\hline Cruciferous vegetables & - & 0.54 & - \\
\hline Other fruit & - & 0.59 & - \\
\hline Other greens & - & 0.60 & -0.22 \\
\hline Tomato sauce & - & 0.50 & - \\
\hline Other vegetables & 0.22 & 0.54 & - \\
\hline Beans, peas & 0.15 & 0.25 & - \\
\hline Pickled vegetables & 0.15 & 0.26 & 0.15 \\
\hline Total cereals and grains & 0.23 & 0.38 & 0.28 \\
\hline Whole grains & - & 0.33 & - \\
\hline Citrus & - & 0.34 & - \\
\hline Berries & - & 0.45 & - \\
\hline Dried fruit & - & 0.39 & - \\
\hline Vegetable juice & - & 0.17 & - \\
\hline Beer & 0.19 & - & - \\
\hline White wine & - & - & - \\
\hline Red wine & - & - & - \\
\hline Liquor & - & - & - \\
\hline Desserts and sweets & 0.31 & - & 0.63 \\
\hline Pies, tarts & 0.15 & - & 0.54 \\
\hline Canned fruit & - & 0.21 & 0.23 \\
\hline Jam, jelly & - & - & 0.26 \\
\hline Proportion of VAR explained (\%) & 39.79 & 22.93 & 11.10 \\
\hline Cumulative VAR explained (\%) & 39.79 & 62.72 & 73.82 \\
\hline
\end{tabular}

\section{Dietary patterns and cancer recurrence or death}

The highest quartile of processed meat pattern was significantly associated with poorer DFS after the adjustment for other predictors of CRC recurrence and death (HR 1.82, $95 \%$ CI 1.07 to 3.09 ), although no overall trend was observed in the HRs across the whole distribution of factor scores ( $p$ for trend $=0.09$ ) (table 3). Nevertheless, neither the prudent vegetable pattern nor the high-sugar pattern was observed to be significantly associated with predefined patient outcomes (ie, DFS and OS).

When stratified by tumour site, however, the association between processed meat pattern and DFS remained statistically significant only for patients who had tumours located in the colon (the highest vs the lowest quartile, HR 2.29, 95\% CI 1.19 to 4.40 ) and not the rectum (HR $0.97,95 \%$ CI 0.38 to 2.45 ). Similarly, when OS was the outcome, the positive association between increasing consumption of the processed meat pattern and mortality was restricted to patients whose tumours were diagnosed in the colon (the fourth vs first quartiles: HR $2.13,95 \%$ CI 1.03 to 4.43 ).

In the stratified analyses for dietary patterns, there was evidence for effect modification by sex $(p=0.04)$ for the association of processed meat pattern with DFS (HR 3.85 for women and 1.22 for men) (table 4). However, no evidence was observed to suggest that the effects of 
Table 2 Baseline characteristics of $529 \mathrm{CRC}$ patients by quartiles of the three major dietary patterns

\begin{tabular}{|c|c|c|c|c|c|c|c|c|c|c|c|c|c|c|c|}
\hline & \multicolumn{5}{|c|}{ Processed meat pattern } & \multicolumn{5}{|c|}{ Prudent vegetable pattern } & \multicolumn{5}{|c|}{ High-sugar pattern } \\
\hline & Q1 (n=132) & Q2 (n=132) & Q3 $(n=133)$ & Q4 (n=132) & p Value ${ }^{\star}$ & Q1 (n=132) & Q2 (n=132) & Q3 $(n=133)$ & Q4 (n=132) & p Value ${ }^{*}$ & Q1 $(n=132)$ & Q2 (n=132) & Q3 $(n=133)$ & Q4 (n=132) & p Value ${ }^{*}$ \\
\hline Age at diagnosis $\dagger$ & $61.4 \pm 8.7$ & $60.6 \pm 9.0$ & $60.2 \pm 8.8$ & $59.3 \pm 9.3$ & 0.29 & $57.4 \pm 10.3$ & $60.1 \pm 7.9$ & $61.0 \pm 9.0$ & $62.1 \pm 8.0$ & $<.0001$ & $59.5 \pm 9.3$ & $60.2 \pm 9.1$ & $60.0 \pm 8.8$ & $61.7 \pm 8.6$ & 0.21 \\
\hline \multicolumn{16}{|l|}{ Sext } \\
\hline Female & $67(50.8)$ & $66(50.0)$ & $39(29.3)$ & $39(29.6)$ & & $38(28.8)$ & $39(29.5)$ & $58(43.6)$ & $76(57.6)$ & & $60(45.5)$ & $49(37.1)$ & $51(38.3)$ & $51(38.6)$ & \\
\hline Male & 65 (49.2) & $66(50.0)$ & $94(70.7)$ & $93(70.5)$ & $<.0001$ & $94(71.2)$ & $93(70.5)$ & 75 (56.4) & 56 (42.4) & $<.0001$ & $72(54.5)$ & 83 (62.9) & $82(61.7)$ & $81(61.4)$ & 0.50 \\
\hline \multicolumn{16}{|l|}{ Stage at diagnosis } \\
\hline $1 / 11$ & $87(65.9)$ & $81(61.4)$ & $70(52.6)$ & $71(53.8)$ & & $72(54.5)$ & $71(53.8)$ & $83(62.4)$ & $83(62.9)$ & & $79(59.8)$ & 77 (58.3) & 77 (57.9) & $76(57.6)$ & \\
\hline \multirow{2}{*}{\multicolumn{16}{|c|}{$\operatorname{BMl}\left(\mathrm{kg} / \mathrm{m}^{2}\right)$}} \\
\hline & & & & & & & & & & & & & & & \\
\hline$<25.0$ & $38(30.6)$ & $47(36.1)$ & $35(26.5)$ & $27(21.1)$ & & $42(33.6)$ & $32(24.8)$ & $34(26.4)$ & $38(29.7)$ & & $33(25.6)$ & $40(31.0)$ & $36(28.1)$ & $38(29.7)$ & \\
\hline $25.0-29.9$ & $57(46.0)$ & $52(40.0)$ & $53(40.2)$ & $53(41.4)$ & & $45((35.2)$ & $57(44.2)$ & $55(42.6)$ & $58(45.3)$ & & $55(42.6)$ & 47 (36.4) & $58(45.3)$ & $55(43.0)$ & \\
\hline$\geq 30$ & 29 (23.4) & $31(23.9)$ & $44(33.3)$ & $48(37.5)$ & 0.03 & $40(31.2)$ & $40(31.0)$ & $40(31.0)$ & $32(25.0)$ & 0.78 & $41(31.8)$ & $42(32.6)$ & 34 (26.6) & 35 (27.3) & 0.63 \\
\hline \multicolumn{16}{|l|}{ Physical activity } \\
\hline$<24.9 \mathrm{MET}$ h/week & $73(55.3)$ & $71(53.4)$ & $56(42.1)$ & $65(49.2)$ & & $68(51.5)$ & $60(45.4)$ & 69 (51.9) & $68(51.5)$ & & $68(51.5)$ & $71(53.8)$ & 69 (51.9) & $57(43.2)$ & \\
\hline$\geq 24.9 \mathrm{MET} \mathrm{h} /$ week & $59(44.7)$ & $61(46.6)$ & $77(57.9)$ & $67(50.8)$ & 0.13 & $64(48.5)$ & $72(54.6)$ & $64(48.1)$ & $64(48.5)$ & 0.67 & $64(48.5)$ & $61(46.2)$ & $64(48.1)$ & $75(56.8)$ & 0.32 \\
\hline \multicolumn{16}{|l|}{ Marital status } \\
\hline Single & $31(23.5)$ & $29(22.0)$ & $18(13.5)$ & $37(28.0)$ & & $26(19.7)$ & $27(20.4)$ & $27(20.3)$ & $35(26.5)$ & & $26(19.7)$ & $30(22.7)$ & 30 (22.6) & $29(22.0)$ & \\
\hline \multirow{2}{*}{\multicolumn{16}{|c|}{ Smoking status }} \\
\hline & & & & & & & & & & & & & & & \\
\hline Ever & 77 (58.3) & $94(71.2)$ & $113(85.0)$ & $104(78.8)$ & & $108(81.8)$ & $97(73.5)$ & $100(75.2)$ & $83(62.9)$ & & $101(76.5)$ & $95(72.0)$ & $95(71.4)$ & $97(73.5)$ & \\
\hline Never & $55(41.7)$ & $38(28.8)$ & $20(15.0)$ & $28(21.2)$ & $<.0001$ & $24(18.2)$ & 35 (26.5) & $33(24.8)$ & 49 (37.1) & 0.006 & $31(23.5)$ & $37(28.0)$ & $38(28.6)$ & $35(26.5)$ & 0.79 \\
\hline \multicolumn{16}{|l|}{ Tumour location } \\
\hline Colon & $91(69.5)$ & $90(68.2)$ & $85(63.9)$ & $79(59.9)$ & & $75(56.8)$ & $91(69.5)$ & $87(65.4)$ & $92(69.7)$ & & $82(62.1)$ & $85(64.9)$ & $87(65.4)$ & $91(68.9)$ & \\
\hline Rectum & $40(30.5)$ & $42(31.8)$ & $48(36.1)$ & $53(40.1)$ & 0.34 & $57(43.2)$ & $40(30.5)$ & 46 (34.6) & $40(30.3)$ & 0.10 & $50(37.9)$ & $46(35.1)$ & 46 (34.6) & $41(31.1)$ & 0.71 \\
\hline \multicolumn{16}{|l|}{ Reported chemoradiotherapy } \\
\hline Yes & $36(27.3)$ & $31(23.5)$ & $20(15.0)$ & $21(15.9)$ & & $24(18.2)$ & $23(17.4)$ & $24(18.1)$ & $37(28.0)$ & & $30(22.7)$ & $28(21.2)$ & $25(18.8)$ & 25 (18.9) & \\
\hline No & 96 (72.7) & $101(76.5)$ & $113(85.0)$ & $111(84.1)$ & 0.04 & $108(81.8)$ & $109(82.6)$ & 109 (81.9) & $95(72.0)$ & 0.10 & $102(77.3)$ & $104(78.8)$ & $108(81.2)$ & $107(81.1)$ & 0.83 \\
\hline \multicolumn{16}{|l|}{ MSI status } \\
\hline MSS/MSI-L & $108(86.4)$ & $110(86.6)$ & $113(91.9)$ & $106(86.9)$ & & $107(85.6)$ & $104(86.7)$ & $113(91.1)$ & $113(88.3)$ & & $107(84.9)$ & $106(87.6)$ & $110(88.0)$ & $114(91.2)$ & \\
\hline MSI-H & 17 (13.6) & $17(13.4)$ & $10(8.1)$ & $16(13.1)$ & 0.49 & $18(14.4)$ & $16(13.3)$ & $11(8.9)$ & $15(11.7)$ & 0.57 & $19(15.1)$ & 15 (12.4) & $15(12.0)$ & $11(8.8)$ & 0.50 \\
\hline \multicolumn{16}{|l|}{$B R A F$ mutation status } \\
\hline Wild type & $104(85.2)$ & 107 (89.9) & $109(90.8)$ & $106(93.0)$ & & $108(91.5)$ & $103(87.3)$ & $112(95.7)$ & $103(84.4)$ & & $103(88.8)$ & $110(91.7)$ & $106(89.1)$ & $107(89.2)$ & \\
\hline V600E mutant & $18(14.8)$ & $12(10.1)$ & $11(9.2)$ & $8(7.0)$ & 0.25 & $10(8.5)$ & 15 (12.7) & $5(4.3)$ & $19(15.6)$ & 0.02 & $13(11.2)$ & $10(8.3)$ & $13(10.9)$ & $13(10.8)$ & 0.88 \\
\hline
\end{tabular}


Table 3 Hazard rate ratios associated with disease-free and overall colorectal cancer survival for quartiles of dietary patterns

\begin{tabular}{|c|c|c|c|c|c|c|c|c|}
\hline & \multicolumn{4}{|c|}{ Disease-free survival } & \multicolumn{4}{|l|}{ Overall survival } \\
\hline & $\begin{array}{l}\text { Number of } \\
\text { events }{ }^{\star} / \text { Number } \\
\text { at risk }\end{array}$ & $\begin{array}{l}\text { Overall CRC } \\
\text { HR }(95 \% \mathrm{CI}) \dagger\end{array}$ & $\begin{array}{l}\text { Colon cancer } \\
\text { HR }(95 \% \mathrm{Cl}) \dagger \\
\end{array}$ & $\begin{array}{l}\text { Rectal cancer } \\
\text { HR }(95 \% \mathrm{Cl}) \dagger\end{array}$ & $\begin{array}{l}\text { Number of } \\
\text { events*/Number } \\
\text { at risk }\end{array}$ & $\begin{array}{l}\text { Overall CRC } \\
\text { HR }(95 \% \text { CI) } \dagger\end{array}$ & $\begin{array}{l}\text { Colon cancer } \\
\mathrm{HR}(95 \% \mathrm{Cl}) \dagger \\
\end{array}$ & $\begin{array}{l}\text { Rectal cancer } \\
\text { HR (95\% Cl)† }\end{array}$ \\
\hline \multicolumn{9}{|c|}{ Processed meat pattern } \\
\hline Q1 & $38 / 132$ & 1.00 & 1.00 & 1.00 & $33 / 132$ & 1.00 & 1.00 & 1.00 \\
\hline Q2 & 45/132 & $1.51(0.95$ to 2.41$)$ & 1.69 (0.97 to 2.96$)$ & 0.91 (0.39 to 2.14$)$ & $40 / 132$ & 1.47 (0.89 to 2.44$)$ & 2.18 (1.16 to 4.09$)$ & $0.75(0.28$ to 2.03$)$ \\
\hline Q3 & $58 / 132$ & 1.56 (0.97 to 2.49$)$ & 1.37 (0.76 to 2.48$)$ & $1.72(0.85$ to 3.95$)$ & $49 / 133$ & 1.32 (0.78 to 2.22$)$ & 1.44 (0.74 to 2.79$)$ & $1.54(0.57$ to 4.13$)$ \\
\hline Q4 & $57 / 132$ & 1.82 (1.07 to 3.09$)$ & 2.29 (1.19 to 4.40$)$ & 0.97 (0.38 to 2.45$)$ & $46 / 132$ & $1.53(0.85$ to 2.74$)$ & 2.13 (1.03 to 4.43$)$ & $1.17(0.41$ to 3.36$)$ \\
\hline$p V$ & alue for trend $\ddagger$ & 0.09 & 0.12 & 0.91 & & 0.25 & 0.40 & 0.59 \\
\hline \multicolumn{9}{|c|}{ Prudent vegetable pattern } \\
\hline Q1 & $46 / 132$ & 1.00 & 1.00 & 1.00 & $41 / 132$ & 1.00 & 1.00 & 1.00 \\
\hline Q2 & $54 / 132$ & $1.21(0.79$ to 1.85$)$ & 1.35 (0.78 to 2.34$)$ & 0.97 (0.47 to 2.01$)$ & $45 / 132$ & 1.09 (0.69 to 1.73$)$ & 1.18 (0.65 to 2.14$)$ & $0.90(0.41$ to 1.98$)$ \\
\hline Q3 & $50 / 133$ & $1.18(0.75$ to 1.86$)$ & $1.16(0.63$ to 2.13$)$ & $1.30(0.65$ to 2.60$)$ & $40 / 133$ & $0.82(0.49$ to 1.36$)$ & 1.04 (0.55 to 1.97$)$ & $0.59(0.25$ to 1.42$)$ \\
\hline Q4 & $48 / 131$ & $1.12(0.69$ to 1.84$)$ & 1.02 (0.52 to 1.99$)$ & 1.28 (0.58 to 2.83$)$ & $42 / 132$ & $1.03(0.61$ to 1.75$)$ & $0.96(0.47$ to 1.96$)$ & $1.00(0.42$ to 2.40$)$ \\
\hline$p V_{c}$ & lue for trend $\ddagger$ & 0.62 & 0.83 & 0.19 & & 0.90 & 0.60 & 0.92 \\
\hline \multicolumn{9}{|c|}{ High-sugar pattern } \\
\hline Q1 & $42 / 131$ & 1.00 & 1.00 & 1.00 & 30.132 & 1.00 & 1.00 & 1.00 \\
\hline Q2 & $54 / 132$ & $1.07(0.70$ to 1.63$)$ & $0.96(0.54$ to 1.68$)$ & 1.30 (0.64 to 2.65$)$ & $48 / 132$ & 1.25 (0.77 to 2.04$)$ & 1.21 (0.62 to 2.36$)$ & $2.12(0.87$ to 5.14$)$ \\
\hline Q3 & $54 / 133$ & 1.09 (0.69 to 1.73$)$ & 0.94 (0.51 to 1.73$)$ & 1.44 (0.67 to 3.07$)$ & $50 / 133$ & 1.64 (0.98 to 2.75$)$ & 1.35 (0.66 to 2.78$)$ & $2.49(1.02$ to 6.10$)$ \\
\hline Q4 & $48 / 132$ & $1.02(0.62$ to 1.69$)$ & $0.99(0.52$ to 1.89$)$ & 1.49 (0.61 to 3.63$)$ & $40 / 132$ & $1.27(0.72$ to 2.25$)$ & $1.16(0.54$ to 2.47$)$ & $1.68(0.55$ to 5.08$)$ \\
\hline $\mathrm{p} \mathrm{V}$ & alue for trend $\ddagger$ & 0.89 & 0.90 & 0.11 & & 0.52 & 0.56 & 0.64 \\
\hline
\end{tabular}

*Events are defined as death/recurrence/metastasis (which occurred earliest) for disease-free survival and deaths for overall survival.

†Cox proportional hazard model adjusted for total energy intake, sex, age at diagnosis, stage at diagnosis, marital status, family history, reported screening procedure, reported

chemoradiotherapy and microsatellite instabilit status, where appropriate.

$\ddagger$ Two-sided $p$ value for test of linear trend was calculated by modelling median values for each quartile of dietary pattern scores as an ordinal variable.

$\mathrm{CRC}$, colorectal cancer; HR, hazard rate ratios. 
Table 4 Disease-free colorectal cancer survival in relation to quartiles of dietary patterns by selected lifestyle and tumour characteristics

\begin{tabular}{|c|c|c|c|c|c|c|c|}
\hline & \multirow[b]{2}{*}{$\begin{array}{l}\text { Number of } \\
\text { events } / \text { no at risk }\end{array}$} & \multicolumn{4}{|c|}{ Quartiles HR (95\% Cl)† } & \multirow[b]{2}{*}{$\begin{array}{l}p \text { Value for } \\
\text { trend } ¥\end{array}$} & \multirow[b]{2}{*}{$\begin{array}{l}\text { p Value for } \\
\text { interactions }\end{array}$} \\
\hline & & Q1 & Q2† & Q3 & Q4 & & \\
\hline \multicolumn{8}{|l|}{ Processed meat pattern } \\
\hline \multicolumn{8}{|l|}{ Sex } \\
\hline Female & $65 / 210$ & 1.00 & 2.20 (0.99 to 4.91$)$ & 2.38 (0.97 to 5.85$)$ & 3.85 (1.49 to 9.99$)$ & 0.03 & \\
\hline Male & $133 / 318$ & 1.00 & 1.20 (0.66 to 2.18$)$ & 1.23 (0.69 to 2.17$)$ & 1.22 (0.64 to 2.32$)$ & 0.27 & 0.04 \\
\hline \multicolumn{8}{|l|}{ Physical activity } \\
\hline$<24.9 \mathrm{MET}$ h/week & $97 / 263$ & 1.00 & 1.96 (1.05 to 3.67$)$ & $2.13(1.11$ to 4.11$)$ & $2.03(0.96-4.30)$ & 0.42 & \\
\hline$\geq 24.9 \mathrm{MET}$ h/week & $101 / 264$ & 1.00 & $1.22(0.59-2.55)$ & $1.27(0.62-2.62)$ & $1.64(0.74-3.62)$ & 0.01 & 0.64 \\
\hline \multicolumn{8}{|l|}{$B R A F$ mutation status } \\
\hline Wild type & $163 / 425$ & 1.00 & 1.28 (0.77 to 2.12$)$ & 1.41 (0.80 to 2.34$)$ & $1.80(1.01$ to 3.21$)$ & 0.009 & \\
\hline V600E mutant & $17 / 49$ & 1.00 & $1.82(0.40$ to 8.34$)$ & $0.54(0.10$ to 2.83$)$ & 0.79 (0.09 to 7.01$)$ & 0.50 & 0.80 \\
\hline \multicolumn{8}{|c|}{ Prudent vegetables pattern } \\
\hline \multicolumn{8}{|l|}{ Sex } \\
\hline Female & $65 / 210$ & 1.00 & 1.57 (0.59 to 4.20$)$ & 1.55 (0.63 to 3.85$)$ & $1.22(0.46$ to 3.24$)$ & 0.71 & \\
\hline Male & $133 / 318$ & 1.00 & 1.25 (0.76 to 2.04$)$ & $1.08(0.62$ to 1.88$)$ & 1.14 (0.62 to 2.09$)$ & 0.67 & 0.65 \\
\hline \multicolumn{8}{|l|}{ Physical activity } \\
\hline$<24.9 \mathrm{MET}$ h/week & $97 / 263$ & 1.00 & $1.48(0.80$ to 2.76$)$ & 1.52 (0.81 to 2.87$)$ & 1.22 (0.56 to 2.69$)$ & 0.66 & \\
\hline$\geq 24.9$ MET h/week & $101 / 264$ & 1.00 & $1.02(0.55$ to 1.89$)$ & $1.02(0.53$ to 1.96$)$ & 1.05 (0.55 to 2.04$)$ & 0.03 & 0.83 \\
\hline \multicolumn{8}{|l|}{$B R A F$ mutation status } \\
\hline Wild type & $163 / 425$ & 1.00 & 1.32 (0.83 to 2.10$)$ & $1.29(0.80$ to 2.08$)$ & 1.19 (0.70 to 2.02$)$ & 0.58 & \\
\hline V600E mutant & $17 / 49$ & 1.00 & $2.50(0.38$ to 16.59$)$ & $0.88(0.06$ to 12.99$)$ & $1.24(0.12$ to 13.20$)$ & 0.73 & 0.80 \\
\hline \multicolumn{8}{|l|}{ High-sugar pattern } \\
\hline \multicolumn{8}{|l|}{ Sex } \\
\hline Female & $65 / 210$ & 1.00 & $1.41(0.63$ to 3.16$)$ & 0.88 (0.36 to 2.15$)$ & 0.82 (0.30 to 2.27$)$ & 0.42 & \\
\hline Male & $133 / 318$ & 1.00 & 1.14 (0.67 to 1.97$)$ & 1.34 (0.75 to 2.39$)$ & 1.39 (0.73 to 2.66$)$ & 0.06 & 0.72 \\
\hline \multicolumn{8}{|l|}{ Physical activity } \\
\hline <24.9 MET h/week & $97 / 263$ & 1.00 & 1.01 (0.55 to 1.86$)$ & $1.10(0.56$ to 2.16$)$ & 1.19 (0.56 to 2.54$)$ & 0.06 & \\
\hline$\geq 24.9 \mathrm{MET}$ h/week & $101 / 264$ & 1.00 & $1.36(0.70$ to 2.65$)$ & 1.21 (0.60 to 2.45$)$ & 1.04 (0.49 to 2.22$)$ & 0.86 & 0.26 \\
\hline \multicolumn{8}{|l|}{$B R A F$ mutation status } \\
\hline Wild type & $163 / 425$ & 1.00 & 0.99 (0.61 to 1.59$)$ & 1.20 (0.71 to 2.01$)$ & 1.03 (0.59 to 1.82$)$ & 0.70 & \\
\hline V600E mutant & $17 / 49$ & 1.00 & $0.53(0.07$ to 4.25$)$ & 0.27 (0.04 to 1.66$)$ & 0.32 (0.04 to 2.64$)$ & 0.09 & 0.33 \\
\hline
\end{tabular}

*Events are defined as death/recurrence/metastasis (which occurred earliest) for disease-free survival and deaths for overall survival.

†Cox proportional hazard model adjusted for total energy intake, sex, age at diagnosis, stage at diagnosis, body mass index, marital status, family history, reported screening procedure, reported chemoradiotherapy and MSI status, where appropriate.

†Two-sided $p$ Value for test of linear trend was calculated by modelling median values for each quartile of dietary pattern scores as an ordinal variable.

$\S p$ Value for interaction is the significance of interaction term between smoking and respective stratification variable, calculated from Wald test.

$\mathrm{HR}$, hazard rate ratio; METh/week, metabolic equivalent hours per week. 
other dietary patterns on cancer recurrence or death were modified by physical activity, BRAF mutation status and MSI (data not shown).

In the sensitivity analysis, when advanced-stage patients who died before admittance were excluded, the association between processed meat pattern and survival among CRC patients remained significant.

\section{DISCUSSION}

Three dietary patterns, termed 'processed meat pattern', 'prudent vegetable pattern' and 'high-sugar pattern', were generated in this cohort study. We found that high conformity with the processed meat pattern, characterised by high intakes of processed meat, red meat, fish and processed fish, is associated with decreased DFS of CRC, specifically of colon cancer. The differential associations by subsite indicate disease heterogeneity. On the contrary, increasing consumption of the prudent vegetable pattern and the high-sugar pattern displayed no clear relationships with mortality or recurrence.

The processed meat pattern in the present study shares most characteristics of the Western diet referred to in previous studies on CRC risk, which indicates a positive association between the Western dietary pattern and CRC risk. ${ }^{7}{ }^{9}$ However, there has been minimal research examining the association between dietary factors (eg, nutrient, carbohydrate, protein and lipid intake) and survival of CRC patients; ${ }^{30} 31$ moreover, our literature review identified only one study that investigated the relationship between dietary patterns and survival among CRC patients. Consistent with our results, that prospective cohort study of 1009 stage III colon cancer patients ${ }^{9}$ reported a deleterious disease-free colon cancer prognosis for patients reporting high levels of Western dietary pattern intake.

The mechanisms explaining the impact of red and processed meat on CRC mortality are still unclear; however, some biological mechanisms that link diet factors to CRC risk may continue after diagnosis and subsequently impact cancer progression and survival. ${ }^{32}$ For example, strong carcinogens such as $\mathrm{N}$-nitroso compounds (NOCs) and probable carcinogenic mutagens like heterocyclic amines and polycylic aromatic hydrocarbons, which have been suggested as significant contributors for CRC development, ${ }^{33} 34$ are found in smoked, fried or high-temperature cooked meat. Sandhu et $a l^{35}$ reported that a Western dietary pattern is related to high levels of serum insulin and insulin-like growth factors, and these hormones are found to be associated with tumour growth and the inhibition of apoptosis. In addition, a growing body of evidence suggests that disruption of the normal gut microflora is associated with human disease, including the pathogenesis of the intestinal tract (eg, inflammatory bowel disease) and other diseases such as obesity, cardiovascular disease and autoimmune conditions. ${ }^{36}{ }^{37}$ Alterations in intestinal microbiota are also strongly associated with colonic polyp formation and with the risk of developing CRC. ${ }^{38}$ Given the major role of diet on the intestinal microbiome, ${ }^{39}$ our findings between dietary patterns and CRC survival may also be explained by the impact of dietary patterns on gut microflora and health outcomes.

The influence of processed meat pattern on survival was evident among women rather than men in our study. Previous studies revealed that higher colon $\mathrm{pH}$ and longer intestine transit time in women compared with men can influence the production of secondary bile acid or NOCs, ${ }^{40}$ resulting in gender differences in CRC development. This is the first study that considered effect modifications between dietary patterns and tumour molecular phenotype (ie, BRAF mutation) on CRC survival. BRAF mutation is found to be significantly associated with poor CRC survival; ${ }^{41}$ however, whether it can modify the impact of dietary factors on CRC survival is not known. Although stratified analyses in our study demonstrated a processed meat diet to significantly decrease survival time only in patients with $B R A F$ wild type tumour, no evident interactions were detected. Further research is clearly warranted to verify these findings and to determine the biological pathways that rationalise the underlying interactions between diet and tumour molecular features.

A reasonably large sample size with detailed information of patients is a merit of our study. These data not only include demographic and personal lifestyle information, but also some molecular characteristics obtained from genetic testing. The ample information enables us to perform stratification analysis to control and assess effect modifiers and confounders. Several limitations of this study should be recognised. First, the results may be skewed by recall bias since the participants recalled their food consumption from 1 year prior to CRC diagnosis; however, this non-differential misclassification is only expected to bias the results towards the null. Second, dietary patterns in this study only reflect food consumption before diagnosis; it is unknown whether participants modified their diet post diagnosis. Since previous research has shown minimal change in diet between prediagnosis and postdiagnosis among cancer patients, ${ }^{31}$ the current study did not examine dietary changes before and after diagnosis. Moreover, immortal persontime bias may impact results. However, this is minimised by using proxies to enrol deceased patients.

In summary, we found that high conformity to the processed meat pattern is significantly associated with an increased risk of all-cause mortality and recurrence of CRC. Though our study did not find a difference in effect by tumour molecular phenotype, larger molecular studies should be conducted to examine if such differences exist. Ultimately, confirmation of these findings and the underlying mechanisms await further studies. Our observation not only underlines the importance of maintaining a healthy diet, but also provides guidance to efficacious dietary interventions, ${ }^{8}$ that is, people may lower their risk of CRC mortality by reducing consumption of a processed meat pattern diet. 
Author affiliations

${ }^{1}$ Division of Community Health and Humanities, Faculty of Medicine, Memorial University of Newfoundland, St. John's, Newfoundland, Canada ${ }^{2}$ Department of Epidemiology, School of Public Health, Tianjin Medical University, Tianjin, China

${ }^{3}$ Discipline of Genetics, Faculty of Medicine, Memorial University of Newfoundland, St. John's, Newfoundland, Canada

${ }^{4}$ Discipline of Oncology, Faculty of Medicine, Memorial University of Newfoundland, St. John's, Newfoundland, Canada

${ }^{5}$ Department of Epidemiology, The First Affiliated Hospital of Wenzhou Medical College, Wenzhou, China

${ }^{6}$ Clinical Epidemiology Unit, Faculty of Medicine, Memorial University of Newfoundland, St. John's, Newfoundland, Canada

${ }^{7}$ Prosserman Centre for Health Research, Samuel Lunenfeld Research Institute, Mount Sinai Hospital, Toronto, Ontario, Canada

${ }^{8}$ Epidemiology Research Program, American Cancer Society, Atlanta, Georgia, USA

Acknowledgements This work was supported by the Canadian Institutes of Health Research Team Grant [CIHR-CPT79845] and Canadian Institutes of Health Research Team in Interdisciplinary Research on Colorectal Cancer Studentship [205835]. Yun Zhu and Hao Wu were awarded by the Newfoundland and Labrador Centre for Applied Health Research through Master's fellowship.

Contributors PPW, YZ and HW contributed to the conception and design of this manuscript. YZ and HW analysed the data. YZ, HW, PPW, JW, TW, RJ, ED and PTC drafted and revised the manuscript. SS, RG, MW, BR, SB, JRM and PSP were responsible for data collection. All the authors provided final approval.

Funding This work was supported by the Canadian Institutes of Health Research Team Grant (CIHR-CPT79845) and Canadian Institutes of Health Research Team in Interdisciplinary Research on Colorectal Cancer Studentship (205835). Yun Zhu and Hao Wu were awarded by the Newfoundland and Labrador Centre for Applied Health Research through Master's fellowship.

Competing interests None.

Ethics approval Human Investigation Committee in Memorial University of Newfoundland.

Provenance and peer review Not commissioned; externally peer reviewed.

Data sharing statement No additional data are available.

\section{REFERENCES}

1. Canadian Cancer Statistics 2012. Toronto, ON: Canadian Cancer Society's Steering Committee on Cancer Statistics. Canadian Cancer Society, 2012.

2. Norat T, Chan D, Lau R, et al. WCRF/AICR Systematic Literature Review Continuous Update Project Report. Imperial College London, 2010.

3. Dixon LB, Balder HF, Virtanen MJ, et al. Dietary patterns associated with colon and rectal cancer: results from the Dietary Patterns and Cancer (DIETSCAN) Project. Am J Clin Nutr 2004;80:1003-11.

4. Terry $\mathrm{P}, \mathrm{Hu} \mathrm{FB}$, Hansen $\mathrm{H}$, et al. Prospective study of major dietary patterns and colorectal cancer risk in women. $\mathrm{Am} J$ Epidemiol 2001;154:1143-9.

5. Kwan ML, Weltzien E, Kushi LH, et al. Dietary patterns and breast cancer recurrence and survival among women with early-stage breast cancer. J Clin Oncol 2009;27:919-26.

6. Kim MK, Sasaki S, Otani T, et al. Dietary patterns and subsequent colorectal cancer risk by subsite: a prospective cohort study. Int $J$ Cancer 2005;115:790-8.

7. Slattery ML, Boucher KM, Caan BJ, et al. Eating patterns and risk of colon cancer. Am J Epidemiol 1998;148:4-16.

8. Williams CD, Satia JA, Adair LS, et al. Dietary patterns, food groups, and rectal cancer risk in Whites and African-Americans. Cancer Epidemiol Biomarkers Prev 2009;18:1552-61.

9. Meyerhardt JA, Niedzwiecki D, Hollis D, et al. Association of dietary patterns with cancer recurrence and survival in patients with stage III colon cancer. JAMA 2007;298:754-64.

10. Fung T, Hu FB, Fuchs $\mathrm{C}$, et al. Major dietary patterns and the risk of colorectal cancer in women. Arch Intern Med 2003;163:309-14.
11. Squires J, Roebothan B, Buehler S, et al. Pickled meat consumption and colorectal cancer (CRC): a case-control study in Newfoundland and Labrador, Canada. Cancer Causes Control 2010;21:1513-21.

12. Sun Z, Zhu Y, Wang PP, et al. Reported intake of selected micronutrients and risk of colorectal cancer: results from a large population-based case-control study in Newfoundland, Labrador and Ontario, Canada. Anticancer Res 2012;32:687-96.

13. Sun Z, Liu L, Wang PP, et al. Association of total energy intake and macronutrient consumption with colorectal cancer risk: results from a large population-based case-control study in Newfoundland and Labrador and Ontario, Canada. Nutr J 2012;11:18.

14. Woods MO, Younghusband HB, Parfrey PS, et al. The genetic basis of colorectal cancer in a population-based incident cohort with a high rate of familial disease. Gut 2010;59:1369-77.

15. Green RC, Green JS, Buehler SK, et al. Very high incidence of familial colorectal cancer in Newfoundland: a comparison with Ontario and 13 other population-based studies. Fam Cancer 2007;6:53-62.

16. Stram DO, Hankin JH, Wilkens LR, et al. Calibration of the dietary questionnaire for a multiethnic cohort in Hawaii and Los Angeles. Am J Epidemiol 2000;151:358-70.

17. Sharma S, Iwasaki M, Kunieda C, et al. Development of a quantitative food frequency questionnaire for assessing food, nutrient, and heterocyclic aromatic amines intake in Japanese Brazilians for a colorectal adenoma case-control study. Int J Food Sci Nutr 2009;60(Suppl 7):128-39.

18. Jain MG, Rohan TE, Soskolne CL, et al. Calibration of the dietary questionnaire for the Canadian Study of Diet, Lifestyle and Health cohort. Public Health Nutr 2003;6:79-86.

19. Hankin JH, Wilkens LR, Kolonel LN, et al. Validation of a quantitative diet history method in Hawaii. Am J Epidemiol 1991;133:616-28.

20. Dr. H. Bliss Murphy Cancer Care Foundation. http://www cancercarefoundation.nl.ca/ (accessed 18 Jul 2012).

21. MANUAL of the international statistical classification of diseases, injuries, and causes of death. Addendum 1. Supplementary interpretations and instructions for coding causes of death. Bull World Health Org Suppl 1953;7(Suppl 6):1-55.

22. Raptis S, Mrkonjic M, Green RC, et al. MLH1-93G>A promote polymorphism and the risk of microsatellite-unstable colorectal cancer. J Natl Cancer Inst 2007:99:463-74.

23. Campbell PT, Jacobs ET, Ulrich CM, et al. Case-control study of overweight, obesity, and colorectal cancer risk, overall and by tumor microsatellite instability status. J Natl Cancer Inst 2010;102:391-400.

24. Loughrey MB, Waring PM, Tan A, et al. Incorporation of somatic BRAF mutation testing into an algorithm for the investigation of hereditary non-polyposis colorectal cancer. Fam Cancer 2007:6:301-10.

25. Phipps Al, Baron J, Newcomb PA. Prediagnostic smoking history, alcohol consumption, and colorectal cancer survival: the Seattle Colon Cancer Family Registry. Cancer 2010;117:4948-57

26. Hile SE, Shabashev S, Eckert KA. Tumor-specific microsatellite instability: do distinct mechanisms underlie the MSI-L and EMAST phenotypes? Mutat Res 2012 (In press).

27. Joliffe IT, Morgan BJ. Principal component analysis and exploratory factor analysis. Stat Methods Med Res 1992;1:69-95.

28. Heidemann $\mathrm{C}$, Schulze MB, Franco $\mathrm{OH}$, et al. Dietary patterns and risk of mortality from cardiovascular disease, cancer, and all causes in a prospective cohort of women. Circulation 2008;118:230-7.

29. Introduction to SAS, UCLA: Academic Technology Services, Statistical Consulting Group.http://www.ats.ucla.edu/stat/examples/ asa/test proportionality.htm (accessed 4 Jun 2012)

30. Slattery ML, French TK, Egger MJ, et al. Diet and survival of patients with colon cancer in Utah: is there an association? Int $J$ Epidemiol 1989;18:792-7.

31. Dray X, Boutron-Ruault MC, Bertrais S, et al. Influence of dietary factors on colorectal cancer survival. Gut 2003;52:868-73.

32. Dolecek TA, McCarthy BJ, Joslin CE, et al. Prediagnosis food patterns are associated with length of survival from epithelial ovarian cancer. J Am Diet Assoc 2010;110:369-82

33. Bingham SA, Pignatelli B, Pollock JR, et al. Does increased endogenous formation of $\mathrm{N}$-nitroso compounds in the human colon explain the association between red meat and colon cancer? Carcinogenesis 1996;17:515-23. 
34. Cross AJ, Sinha R. Meat-related mutagens/carcinogens in the etiology of colorectal cancer. Environ Mol Mutagen 2004:44:44-55.

35. Sandhu MS, Dunger DB, Giovannucci EL. Insulin, insulin-like growth factor-I (IGF-I), IGF binding proteins, their biologic interactions, and colorectal cancer. J Natl Cancer Inst 2002;94:972-80.

36. Rabizadeh $\mathrm{S}$, Sears $\mathrm{C}$. New horizons for the infectious diseases specialist: how gut microflora promote health and disease. Curr Infect Dis Rep 2008;10:92-8.

37. Kinross JM, Darzi AW, Nicholson JK. Gut microbiome-host interactions in health and disease. Genome Med 2011;3:14.
38. Tjalsma $\mathrm{H}$, Boleij A, Marchesi JR, et al. A bacterial driver-passenger model for colorectal cancer: beyond the usual suspects. Nat Rev Microbiol 2012;10:575-82.

39. Backhed F, Ley RE, Sonnenburg JL, et al. Host-bacterial mutualism in the human intestine. Science 2005;307:1915-20.

40. Takachi R, Tsubono $\mathrm{Y}, \mathrm{Baba} \mathrm{K}$, et al. Red meat intake may increase the risk of colon cancer in Japanese, a population with relatively low red meat consumption. Asia Pac J Clin Nutr 2011;20:603-12.

41. Safaee Ardekani G, Jafarnejad SM, Tan L, et al. The prognostic value of BRAF mutation in colorectal cancer and melanoma: a systematic review and meta-analysis. PLoS One 2012;7:e47054 